Target article title: Suboptimality in Perceptual Decision Making

Target article authors: Dobromir Rahnev and Rachel N. Denison

\title{
While optimal models are useful, optimality claims are not that common.
}

Claire Chambers, clairenc@seas.upenn.edu

Konrad Paul Kording, kording@seas.upenn.edu

Institution: University of Pennsylvania

Mailing address: Department of Bioengineering and Department of Neuroscience at University of Pennsylvania, Philadelphia, 19104, USA

\begin{abstract}
Rahnev and Denison argue that human behavior is often described as 'optimal', despite many previous findings of suboptimality. We address how the literature handles these concepts and discuss our own findings on suboptimality. While we agree that the field should embrace the 'systematic weirdness of human behavior', this does not detract from the value of the Bayesian approach.
\end{abstract}


Rahnev and Denison (2018) rightly point out a fact endorsed by many prominent Bayesian theorists, that human behavior often deviates from the predictions of statistically optimal models (Acerbi, Vijayakumar, \& Wolpert, 2014; Beck, Ma, Pitkow, Latham, \& Pouget, 2012; Summerfield \& Tsetsos, 2015). Being data scientists, we want to first trace the way the literature handles the concepts discussed by the authors. Specifically, we want to establish if there is a 'current narrow focus on optimality' as argued by Rahnev and Denison. We searched Pubmed for journal articles published between 01/01/1995 and 04/01/2018 that addressed optimality in human behavior. We found that while the number of articles that apply Bayesian modeling to human behavior has increased (Fig. 1), only a small proportion of the articles that we analyzed make optimality or near-optimality claims (.30), and that claims of suboptimality are in roughly equal proportion (.25). Nor have optimality claims drastically increased relative to the overall number of articles that use Bayesian modeling over the last two decades: 2000s (.32), 2010s (.30). The majority of publications that we analyzed use Bayesian modeling as a tool to understand behavior at the computational level and/or emphasize the suboptimality of behavior. Therefore, optimality claims may not be as prevalent as the authors contend.

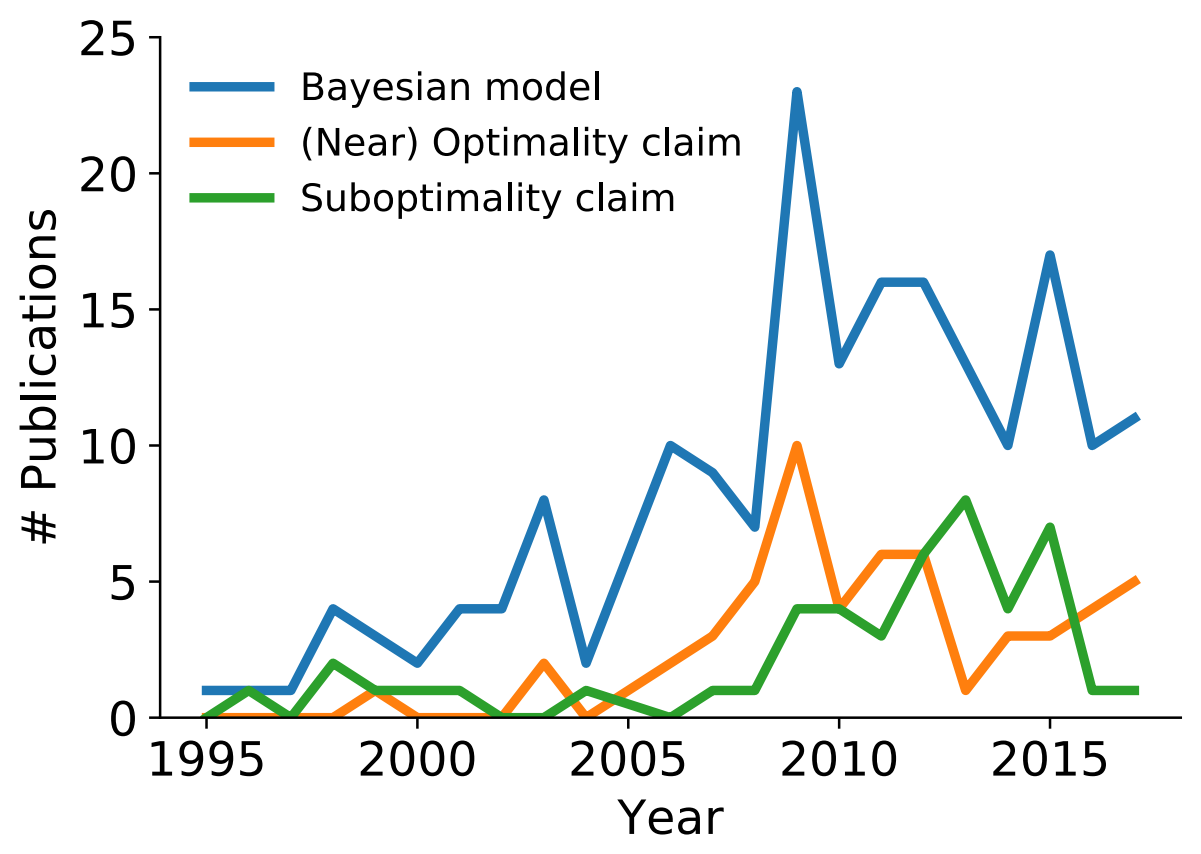

Figure 1. Number of journal articles as a function of year of publication that use Bayesian models to describe behavior, that make (near) optimality claims and that make suboptimality claims. We used the following search terms: (sub)optimality perception, (sub)optimal perceptual decision making, Bayesian psychophysics, Bayesian observer model behavior/perception. We included in our analysis the 185 articles which reported human psychophysics data and modeling. We manually coded the type of the claim made by each paper. 
We endorse the view of Rahnev and Denison that humans deviate from statistically optimal behavior, and some of our recent perceptual work has added new ways in which behavior is suboptimal. For example, prior knowledge acquired in a sensorimotor task does not fully generalize to a perceptual decision-making task that requires the same probabilistic treatment of sensory and prior information (Chambers, Fernandes, \& Kording, 2017). Also, we show that young children fail to incorporate experimentally-imposed priors into their sensorimotor estimates and learn to approximate efficient use of statistical information during development (Chambers, Sokhey, Gaebler-Spira, \& Kording, 2017). If the underlying neural processes are innately 'Bayes optimal', then behavior should reflect this under different conditions and without learning. We have observed this not to be the case when the decision modality changes in decision-making in adults and during child development. These findings underline the importance of assessing the generality of claims concerning the efficient use of statistical information in human decision-making and add to the literature on suboptimal perceptual decision-making discussed by Rahnev and Denison.

Rahnev and Denison advocate for a change in the culture of how behavioral data is modeled. They argue that much existing work is limited because it includes one ad-hoc model for one set of experiments. We agree that this is problematic because based on this ad-hoc approach we cannot know how well optimal models generalize or 'transfer' to other situations (Maloney \& Mamassian, 2009). The authors recommend that in cases of findings of suboptimality, the assumptions of each model component (likelihood, prior, cost function, decision rule) should be examined, and that in cases of good agreement with optimal models, model performance should be examined under different conditions and tasks. We agree with the authors' prescriptions on a more rigorous approach to following up on previous claims and believe that this practice will help the field to develop models that provide more complete accounts of human behavior.

However, it should be emphasized that Bayesian optimal models remain an important tool for building computational models of behavior. Uncertainty exists in the outside world and we do well in everyday tasks by taking uncertainty into account (Kersten, Mamassian, \& Yuille, 2004; Kording \& Wolpert, 2006; Vilares \& Kording, 2011). Alternative non-optimal frameworks, like the 'bag of tricks' (Ramachandran, 1990) mentioned by the authors and heuristic models (Gigerenzer \& Gaissmaier, 2011; Kahneman, Slovic, \& Tversky, 1982) do not contain explicit formulations of how probabilistic information should be combined and do not as easily capture how we deal with uncertainty as Bayesian models do. The Bayesian framework has other advantages as a computational framework. It provides an important benchmark for human performance that can constrain non-optimal models. It is a compact mathematical framework that avoids assumptions about implementational details. It is transparent: the components of the model (prior, likelihood, cost function, decision) must be declared (Griffiths, Chater, Norris, \& Pouget, 2012). While we 
agree that the usefulness of labelling of behavior as 'optimal' is questionable, we maintain that Bayesian modeling is still an important tool for the computational understanding of behavior.

\section{References}

Acerbi, L., Vijayakumar, S., \& Wolpert, D. M. (2014). On the Origins of Suboptimality in Human Probabilistic Inference. PLoS Computational Biology, 10(6), e1003661. https://doi.org/10.1371/journal.pcbi.1003661

Beck, J. M., Ma, W. J., Pitkow, X., Latham, P. E., \& Pouget, A. (2012). Not Noisy, Just Wrong: The Role of Suboptimal Inference in Behavioral Variability. Neuron, 74(1), 30-39. https://doi.org/10.1016/j.neuron.2012.03.016

Chambers, C., Fernandes, H., \& Kording, K. (2017). Policies or Knowledge: Priors differ between perceptual and sensorimotor tasks. BioRxiv, 132829. https://doi.org/https://doi.org/10.1101/132829

Chambers, C., Sokhey, T., Gaebler-Spira, D., \& Kording, K. (2017). The development of Bayesian integration in sensorimotor estimation. BioRxiv, 136267. https://doi.org/http://dx.doi.org/10.1101/136267

Gigerenzer, G., \& Gaissmaier, W. (2011). Heuristic decision making. Annual Review of Psychology, 62, 451-482. https://doi.org/10.1146/annurev-psych-120709-145346

Griffiths, T. L., Chater, N., Norris, D., \& Pouget, A. (2012). How the Bayesians got their beliefs (and what those beliefs actually are): Comment on Bowers and Davis (2012). Psychological Bulletin, 138(3), 415-422. https://doi.org/10.1037/a0026884

Kahneman, D., Slovic, P., \& Tversky, A. (1982). Judgment under uncertainty. Science, 185(4157), 11241131. https://doi.org/10.1093/oxfordhb/9780195376746.013.0038

Kersten, D., Mamassian, P., \& Yuille, A. (2004). Object perception as Bayesian inference. Annual Review of Psychology, 55, 271-304.

Kording, K. P., \& Wolpert, D. M. (2006). Bayesian decision theory in sensorimotor control. Trends in Cognitive Sciences, 10(7), 319-326. https://doi.org/10.1016/j.tics.2006.05.003

Maloney, L. T., \& Mamassian, P. (2009). Bayesian decision theory as a model of human visual perception: testing Bayesian transfer. Visual Neuroscience, 26(1), 147-55. https://doi.org/10.1017/S0952523808080905

Ramachandran, V. (1990). Interactions between motion, depth, color and form: The utilitarian theory of perception. In Vision: Coding and efficiency (pp. 346-360).

Summerfield, C., \& Tsetsos, K. (2015). Do humans make good decisions? Trends in Cognitive Sciences, 19(1), 27-34. https://doi.org/10.1007/s11103-011-9767-z.Plastid 
Vilares, I., \& Kording, K. (2011). Bayesian models: the structure of the world, uncertainty, behaviour, and the brain. Annals of the New York Academy of Sciences, 1224(1), 22-39.

https://doi.org/10.1111/j.1749-6632.2011.05965.x.Bayesian 